\title{
Allele-Specific Detection of SLC22A2 rs316019 Variants Associated with Metformin Disposition through the Kidney
}

\author{
Tasmia Islam Md. Siddiqur Rahman Nilanjana Paul Sharif Akhteruzzaman \\ Abu Ashfaqur Sajib \\ Department of Genetic Engineering and Biotechnology, University of Dhaka, Dhaka, Bangladesh
}

\section{Keywords}

Allele-specific polymerase chain reaction - Diabetes

mellitus · Metformin · OCT2 $\cdot$ rs316019 $\cdot$ SLC22A2

\begin{abstract}
Background: Metformin is prescribed as a first-line drug to treat type 2 diabetes. It is excreted directly and primarily through the SLC22A2 gene-encoded OCT2 transporter in the kidney. rs316019 (c.808G > T, p.270A >S) is the most common variant of SLC22A2, which affects its capacity to clear metformin from the body. Metformin increases the plasma lactate level in a concentration-dependent manner by inhibiting mitochondrial respiration and may lead to a condition known as metformin-associated lactic acidosis (MALA). MALA is a potentially life-threatening complication that can occur within the clinical doses of metformin. Therefore, dose adjustments based on the SLC22A2 rs316019 variants may be beneficial to maximize the efficacy and minimize the toxicity of metformin. Objective: This study was carried out to develop a simple and fast method to define genotype at the rs316019 locus. This method was applied to estimate the rs316019 allele frequencies in the Bangladeshi population. Methods: We designed allele-specific primers to determine
\end{abstract}

genotype at the rs316019 locus using allele-specific polymerase chain reaction (AS-PCR). AS-PCR data were confirmed by targeted sequencing of randomly selected samples. Results: The DNA sequence chromatograms showed the exact genotypes predicted through the AS-PCR method. A proportion of 79.62, 18.01, and $2.37 \%$ of Bangladeshi individuals have GG, GT, and TT genotypes, respectively. Conclusion: We report here a simple and fast method to define genotypes at the rs316019 locus in diabetic patients who are under metformin regimen. Allele frequencies at the rs 316019 locus in the Bangladeshi population are close to those reported in other populations.

(c) 2018 The Author(s)

Published by S. Karger AG, Basel

\section{Introduction}

Diabetes mellitus, commonly referred to as diabetes, is a chronic metabolic disease characterized by hyperglycemia over a prolonged period [1]. The incidence of diabetes is growing in epidemic proportions worldwide. Globally the number of diabetic individuals is speculated to rise from 415 million in 2015 to 642 million in 2040 [2]. Diabetes is a complex condition which affects the entire

Abu Ashfaqur Sajib

Department of Genetic Engineering and Biotechnology University of Dhaka

Dhaka 1000 (Bangladesh)

E-Mail abu.sajib@du.ac.bd 
body. Prolonged hyperglycemia in diabetic patients may result in macro- and microvascular complications leading to a greater risk for heart disease and stroke as well as damage to the nervous system, retina, kidneys, and other organs [3]. The major goal of diabetes treatment, therefore, is to continuously maintain the blood glucose level within the physiological range. Currently this is approached through dietary control, injection of exogenous insulin, and/or a range of oral medications, such as insulin secretagogues and sensitizers [4]. Among the oral medications, metformin is recommended as the first-line treatment in most clinical guidelines on the management of type 2 diabetes, which accounts for nearly $90 \%$ of all cases of the disease $[5,6]$. It is one of the two oral glucoselowering agents in the list of essential medications of the World Health Organization [6].

Metformin reduces hepatic glucose output and increases glucose uptake by the peripheral cells and tissues [7]. Besides, metformin does not affect insulin secretion, unlike the other antihyperglycemic agents [8]. However, concern remains regarding the possible side effect of lactic acidosis, also known as metformin-associated lactic acidosis (MALA) [9]. Lactic acidosis is a condition characterized by serum lactate $>5 \mathrm{mmol} / \mathrm{L}$ and blood $\mathrm{pH}$ $<7.35$ [10]. Though MALA is a relatively rare condition, it is associated with a high mortality rate $(30-50 \%)$, and cases continue to be reported $[9,11]$.

Metformin does not undergo any modification in the liver and is excreted directly in urine through the kidneys [12]. Studies suggest that MALA can occur in patients with even mild renal dysfunction $[9,11]$. An approximately 2- to 4 -fold higher concentration of metformin is observed in the plasma of type 2 diabetic patients with moderate to severe kidney impairment compared to healthy subjects $[9,13]$. Metformin can increase plasma lactate levels in a concentration-dependent manner [9, $14,15]$.

Metformin is excreted primarily through the SLC22A2 gene-encoded OCT2 transporter in the kidney $[16,17]$. SLC22A2 (OCT2) is a member of the SLC22 family of the SLC superfamily. OCT2 transports diverse molecules (including metformin), which remain positively charged under physiological conditions [16-18]. rs316019 (c.808G $>\mathrm{T}, \mathrm{p} .270 \mathrm{~A}>\mathrm{S}$ ) is the most common missense variant of SLC22A2 and has a significant impact on the disposition of metformin [19-24]. While the mechanistic aspect of MALA is still not well defined, recent studies suggested that rs316019 (p.270A $>$ S) polymorphism in SLC22A2 (OCT2) substantially contributes to this complication $[21,23]$. A marked influence of homozygous variant TT genotype was observed on metformin clearance from the body $[14,19,20]$. This particular polymorphism of SLC22A2 should, therefore, be taken into consideration when determining the optimum dosage of metformin.

The International Diabetes Federation has projected that the prevalence of diabetes in Bangladesh will be 13\% by 2030, placing Bangladesh as the 8th largest diabetic populous country in the world [25]. Nearly half of the diagnosed diabetic patients in Bangladesh are treated with metformin alone or in combination with other medicines [26]. There are no Bangladeshi population-specific data on the prevalence of MALA and SLC22A2 rs316019 variants. In this study, we developed a simple and fast allelespecific polymerase chain reaction (AS-PCR)-based method to determine genotype at the rs316019 locus in individuals. We also applied this method to determine the genetic architecture of the Bangladeshi population at the rs316019 locus.

\section{Materials and Methods}

Samples Used in the Study

Buccal swab samples from 125 random Bangladeshi individuals were collected. Briefly, cheek cells from the rinsed and clean mouths of the study subjects were collected using sterile cotton swabs. The cotton swabs were firmly rubbed and rotated along the inside of the cheek for 10-15 s to ensure good contact with the cheek. The swabs were air-dried for a while and carefully placed in collection tubes. The tubes were appropriately labeled and stored at $4{ }^{\circ} \mathrm{C}$ until DNA extraction.

\section{DNA Extraction, Primer Design, and AS-PCR}

DNA was extracted from the buccal cells using the PureLink ${ }^{\mathrm{TM}}$ Genomic DNA Mini Kit (K1820-01; Thermo Fisher Scientific) following the manufacturer's protocol. rs316019 allele-specific primers were designed applying the principle described by Wangkumhang et al. [27]. Primers for glucose-6-phosphate isomerase (GPI) were designed using the Primer-BLAST tool [28]. The specificity of all the primers was checked using the Primer-BLAST tool. Sequences of all primers and other relevant information are given in Table 1. GPI and rs316019 allele-specific sequences were amplified in a duplex PCR in a thermal cycler (Gene Atlas G; Astec Co. Ltd.). An amount of 10-50 ng of genomic DNA template was used for amplification in a final reaction volume of $25 \mu \mathrm{L}$ with $2.5 \mu \mathrm{L}$ of $10 \times$ PCR buffer (EP0702; Thermo Fisher Scientific), $1.0 \mu \mathrm{L}$ of $10 \mathrm{mM}$ dNTP mix (110-002; GeneON), $0.5 \mu \mathrm{L}$ of $10 \mu \mathrm{M}$ rs316019 allele-specific forward primer, $0.5 \mu \mathrm{L}$ of $10 \mu \mathrm{M}$ common reverse primer for $\mathrm{rs} 316019,0.5 \mu \mathrm{L}$ of $10 \mu \mathrm{M}$ GPI forward primer, $0.5 \mu \mathrm{L}$ of $10 \mu \mathrm{M}$ GPI reverse primer, $0.2 \mu \mathrm{L}$ of Taq DNA polymerase (EP0702), and PCR grade water. All primers were purchased from Macrogen Inc. (South Korea). In the negative control PCR, an equal volume of PCR grade water was added instead of genomic DNA. The reaction cycle condition was as follows: an initial denaturation step at $94^{\circ} \mathrm{C}$ for $5 \mathrm{~min}$, then 33 cycles each 
Table 1. Primers used in the study

\begin{tabular}{lllc}
\hline $\begin{array}{l}\text { Combination of } \\
\text { primers in PCR }\end{array}$ & Primer ID & Sequence, 5' $\rightarrow 3^{\prime}$ & $\begin{array}{c}\text { Size, } \\
\text { bp }\end{array}$ \\
\hline $\begin{array}{l}\text { For the G allele } \\
\text { of rs316019 }\end{array}$ & $\begin{array}{l}\text { SLC22A2_rs316019_G_For* } \\
\text { SLC22A2_Rev }\end{array}$ & $\begin{array}{l}\text { GGTGGTTGCAGTTCACAGTCG } \\
\text { ATGCTCTGCTTTCTCCACCC }\end{array}$ & 439 \\
\cline { 2 - 4 } & $\begin{array}{l}\text { GPI_For } \\
\text { GPI_Rev }\end{array}$ & $\begin{array}{l}\text { GGTACACAGGCAAGACCATC } \\
\text { ACCTCCTGAAGAGTATGGCTT }\end{array}$ & 255 \\
\hline $\begin{array}{l}\text { For the T allele } \\
\text { of rs316019 }\end{array}$ & SLC22A2_rs316019_T_For* & GGTGGTTGCAGTTCACAGTCT & 439 \\
& SLC22A2_Rev & ATGCTCTGCTTTCTCCACCC & 255 \\
\cline { 2 - 4 } & GPI_For & GGTACACAGGCAAGACCATC & \\
\hline For sequencing & ACCTCCTGAAGAGTATGGCTT & 798 \\
\hline
\end{tabular}

PCR, polymerase chain reaction. * Allele-specific forward primers.

with denaturation at $94^{\circ} \mathrm{C}$ for $30 \mathrm{~s}$, annealing at $58.5^{\circ} \mathrm{C}$ for $1 \mathrm{~min}$, and elongation at $72{ }^{\circ} \mathrm{C}$ for $25 \mathrm{~s}$ followed by a hold at $4{ }^{\circ} \mathrm{C}$. The PCR amplified sequences were resolved in 1.5\% agarose gel (0710; Amresco ${ }^{\circledR}$ ) using $0.5 \times$ Tris-acetate-EDTA (TAE) buffer along with DNA size markers (300003; GeneON). Amplicons were observed in a gel documentation system (WGD-30; Witeg) following incubation with ethidium bromide (0492; Amresco $\left.{ }^{\mathbb{R}}\right)$ in TAE buffer and photographed with the WiseCapture IIM $^{\mathrm{TM}}$ software. The genotype at the rs316019 locus was determined from the banding pattern in the gel photographs.

\section{Sequencing of SLC22A2 rs316019 Variants}

To confirm the genotype data obtained through AS-PCR, randomly selected DNA samples were amplified by PCR in a thermal cycler (Gene Atlas G) using human SLC22A2 gene-specific primers that encompass the rs316019 locus. An amount of 10-50 ng of genomic DNA template was used for amplification in a final reaction volume of $25 \mu \mathrm{L}$ with $2.5 \mu \mathrm{L}$ of $10 \times$ PCR buffer (EP0702), $1.0 \mu \mathrm{L}$ of $10 \mathrm{mM}$ dNTP mix (110-002), $0.5 \mu \mathrm{L}$ of $10 \mu \mathrm{M}$ forward primer, $0.5 \mu \mathrm{L}$ of $10 \mu \mathrm{M}$ reverse primer, $0.2 \mu \mathrm{L}$ of Taq DNA polymerase (EP0702), and PCR grade water. The reaction cycle condition was as follows: an initial denaturation step at $94^{\circ} \mathrm{C}$ for $5 \mathrm{~min}$, then 33 cycles each with denaturation at $94^{\circ} \mathrm{C}$ for $30 \mathrm{~s}$, annealing at $58.5^{\circ} \mathrm{C}$ for $1 \mathrm{~min}$, and elongation at $72^{\circ} \mathrm{C}$ for $45 \mathrm{~s}$ followed by a final extension at $72^{\circ} \mathrm{C}$ for $5 \mathrm{~min}$ once. The PCR amplified sequences were resolved in 1.0\% agarose gel (0710) and observed in a gel documentation system (WGD-30). PCR products were purified using the FavorPrep ${ }^{\mathrm{TM}}$ GEL/PCR Purification Kit (FAGCK 001; Favorgen Biotech Corp.) following the manufacturer's protocol. The concentration and purity of the purified PCR products were cheeked in a NanoDrop ${ }^{\mathrm{TM}} 2000$ spectrophotometer. Sequences of the purified PCR products were obtained through commercial service from Macrogen Inc. (South Korea). Sequences were analyzed using SnapGene Viewer (GSL Biotech LLC).

\section{Data Analysis}

The SLC22A2 rs316019 allele frequencies in the Bangladeshi population and genotypes of individuals were calculated and presented using the Microsoft Excel and the GraphPad Prism ${ }^{\circledR}$ software.

\section{Results}

Duplex PCR was performed to determine genotype at the SLC22A2 rs316019 locus. Gene-specific primers were used to amplify GPI gene sequence as an internal control in PCR. Figure 1 shows the AS-PCR of the SLC22A2 rs316019 variants.

The allele-specific genotypic data were confirmed by sequencing the region of rs316019 locus of randomly selected samples. Figure 2 shows the sequence chromatograms along with corresponding rs 316019 allele-specific duplex PCR. The DNA sequence chromatograms showed the exact genotype predicted through the AS-PCR method.

Of the 125 participants in this study, 54.81 and $45.19 \%$ were female and male, respectively. In this representative Bangladeshi population, the $\mathrm{G}$ and $\mathrm{T}$ alleles at the rs316019 locus had frequencies of 0.86 and 0.14 , respectively (Fig. 3a). Out of the 125 participants, 95 had homozygous $\mathrm{G}$ (GG) genotype, which comprised $76 \%$ of the studied population (Fig. 3b). The TT genotype, which can lead to hyperlactacidemia in patients undergoing metformin therapy [14], comprised only $3.2 \%$ of the studied population. The 1000 Genomes Browser harbors the whole genome sequence information of 86 Bangladeshi
Islam/Rahman/Paul/Akhteruzzaman/ Sajib 
Fig. 1. AS-PCR of SLC22A2 rs316019 variants. Amplified products were separated in $1.5 \%$ agarose gel in $0.5 \times$ TAE. GPI was used as an internal control. Amplicon sizes of GPI and the rs316019 allele-specific products were 255 and 439 bp, respectively. ASPCR, allele-specific polymerase chain reaction; GPI, glucose-6-phosphate isomerase; TAE, Tris-acetate-EDTA.

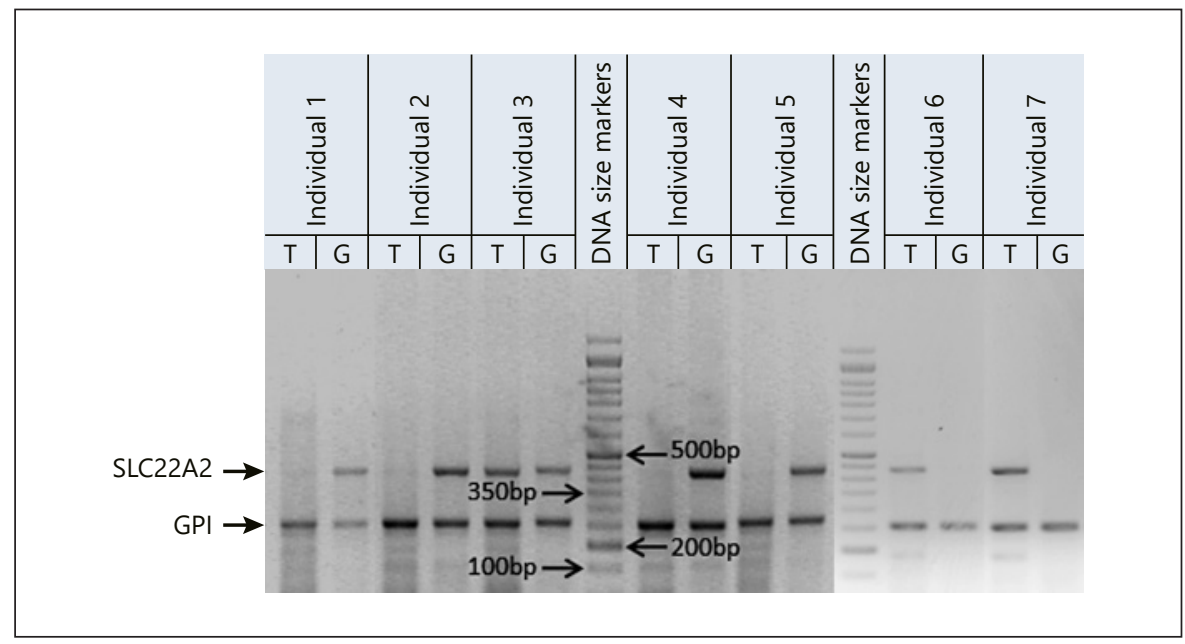

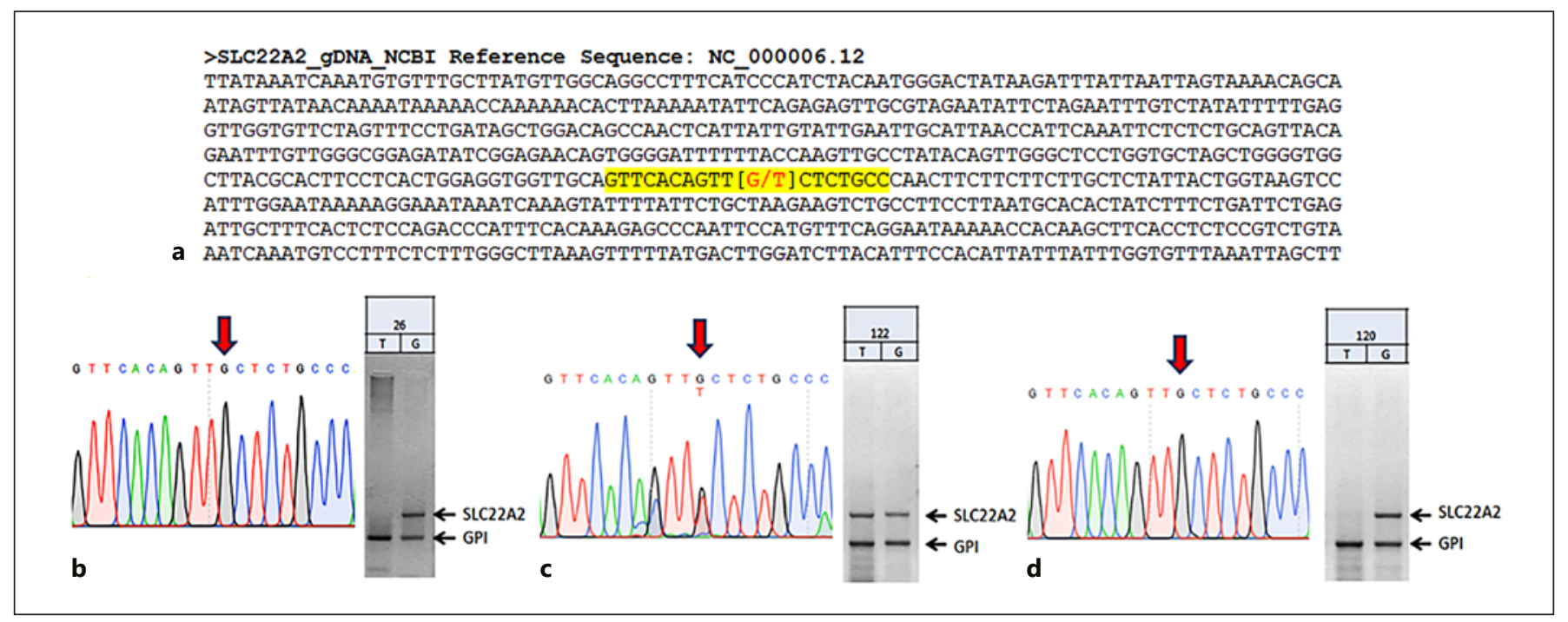

Fig. 2. Targeted sequencing to assess the AS-PCR data. a Partial gene sequence of the human SLC22A2 gene (NCBI reference sequence: NC_000006.12). Sequences shown in the chromatograms below are highlighted in yellow. b-d DNA sequence chromatograms of representative samples alongside the corresponding

individuals [29]. $\mathrm{G}$ and $\mathrm{T}$ allele frequencies in the combined population of 211 individuals (125 and 86 individuals from our study and the 1000 Genomes Browser, respectively) are 0.89 and 0.11 , respectively (Fig. 3a). In these combined population data, 79.62 and $2.37 \%$ of the individuals have GG and TT genotypes, respectively (Fig. 3b). In the combined population, 53.16 and $46.84 \%$ are female and male, respectively. The distribution of rs316019 alleles follows Hardy-Weinberg equilibrium in the Bangladeshi population.

Metformin Disposition: Allele-Specific Detection of rs316019 Variants rs316019 AS-PCR data. A different pair of primers (Table 1) was used in singleplex PCR to amplify the region encompassing the rs316019 site for the sequencing purpose. AS-PCR, allele-specific polymerase chain reaction.

\section{Discussion}

The SLC22A2 gene encoded OCT2 is a polyspecific transporter which transports diverse structurally unrelated cations down their electrochemical gradients across the cell membrane $[16,17]$. Alongside the endogenous metabolites, it transports exogenous compounds, including many drugs which have at least one positively charged moiety at physiological $\mathrm{pH}$ [16-18]. From the perspective that $40 \%$ or more of the prescribed drugs are positively

Int J Diabetes Metab 2018;24:22-28 


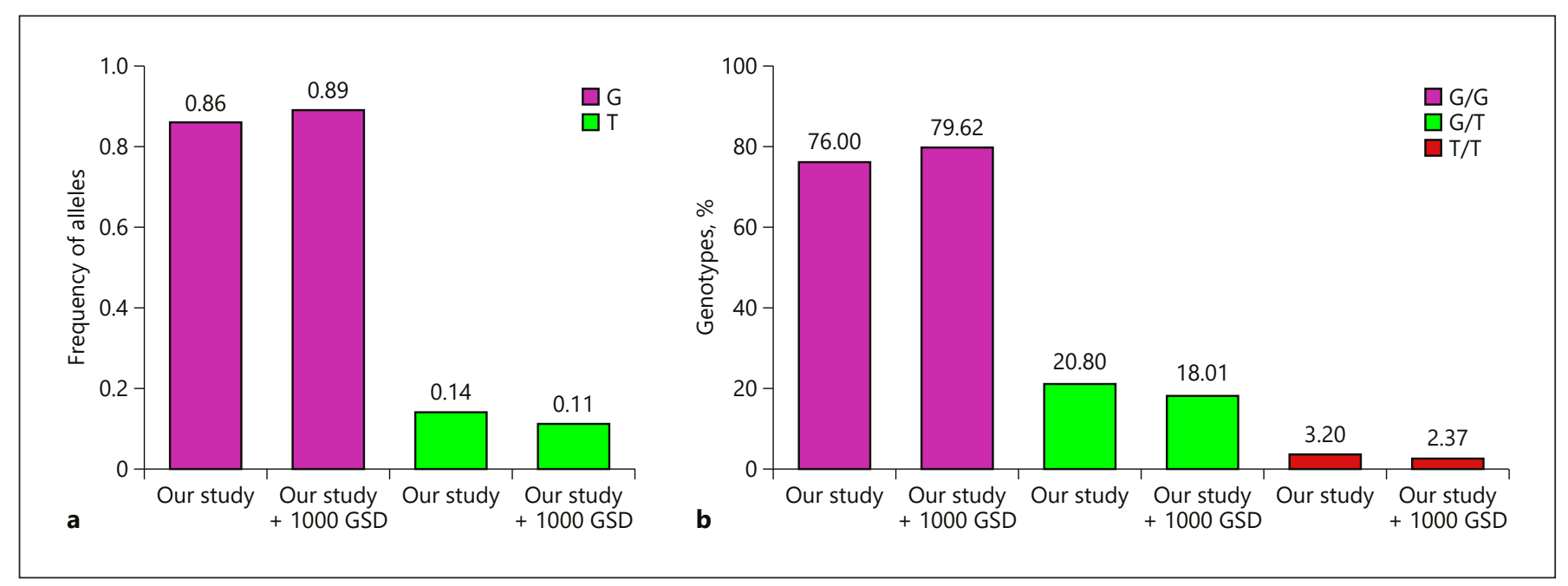

Fig. 3. Genetic architecture of the Bangladeshi population at the SLC22A2 rs 316019 locus. a Frequencies of G and T alleles at the rs316019 locus. b Percentages of different genotypes at the rs316019 locus.

charged, it is apparent that the function of this transporter in the kidney has important pharmacological implications [30]. rs316019 (c.808G $>$ T, p.270A $>$ S) is the most common variant of SLC22A2, which affects its capacity to bind metformin, reduces clearance from circulation, and increases half-life in the body [14, 18-21, 23]. Metformin can increase plasma lactate levels in a concentration-dependent manner by inhibiting mitochondrial respiration, ultimately leading to MALA $[9,14,15]$. MALA is a potentially life-threatening complication which can occur within the clinical doses of metformin $[11,15]$. Preexisting clinical conditions, such as renal impairment, sepsis, anoxia, etc. may make an individual under metformin regimen more prone to MALA $[9,31]$. Therefore, dose adjustments based on the SLC22A2 rs316019 variant may be beneficial to maximize the efficacy and to minimize the toxicity of metformin [32].

We here report a simple and fast AS-PCR-based method to define the genotype of individuals at the SLC22A2 rs316019 locus (Fig. 1). The allele-specific genotypic data showed perfect matching to their corresponding sequence chromatograms (Fig. 2). This simple method can be performed in a basic laboratory setting within $3 \mathrm{~h}$ starting from DNA extraction to allele detection.

The most critical point to ensure in AS-PCR is the avoidance of false-positive amplification as the single nucleotide polymorphism alleles predominantly differ by single base only. The rs316019 allele-specific primers were designed following the principle described by Wangkumhang et al. [27]. In the rs316019 allele-specific prim- ers, one deliberate mismatch at the penultimate base to the rs316019 locus (second to last base at the $3^{\prime}$ end of the primer) was made to introduce a destabilizing effect to eliminate the false-positive amplification. This special feature adds more to the accuracy of alleles-specific primers. The specificity of the primers to the human SLC22A2 gene was checked using the NCBI Primer-BLAST tool as well. To ensure good amplification of the alleles, the annealing temperature of the primers was optimized in thermal gradient PCR.

There is always a possibility of technical error in PCR. Therefore, GPI gene-specific primers were used as an internal control to eliminate false-negative results as well as erroneous interpretation to be homozygous at the rs316019 locus.

Hybridization- or restriction enzyme-based methods are often used to interrogate particular single nucleotide polymorphisms $[33,34]$. One intractable challenge of hybridization-based single nucleotide polymorphism detection is the reduction of cross-hybridization of allelespecific probes, since these differ by a single base [33]. On the other hand, the restriction enzyme-mediated single nucleotide polymorphism detection method is labor intensive, time consuming, relatively more expensive, prone to erroneous result due to incomplete digestion, and difficult to adapt for high-throughput genotyping $[33,35]$. Compared to these, AS-PCR may be considered as a simple and cost-effective method.

By 2040 the number of diabetic individuals in Bangladesh is predicted to rise as high as 13.6 million [36]. Al- 
though metformin is prescribed as a first-line antihyperglycemic drug alone or in combination with other agents to diabetic individuals in Bangladesh [26], there are no Bangladeshi population-specific data on the prevalence of SLC22A2 rs316019 variants. In this study, we applied the AS-PCR method to determine the genetic architecture of the Bangladeshi population at the rs316019 locus. The observed frequency of rs316019 alleles in the Bangladeshi population is similar to those reported in other populations, particularly South Asians (0.87 and 0.13 , respectively) [37].

The AS-PCR-based method described in this study is relatively simple but accurate. This method may be used to detect genotype at the SLC22A2 rs316019 locus in diabetic patients who are under metformin regimen. Dosage adjustments based on the SLC22A 2 rs316019 variant may be beneficial to maximize the efficacy and to minimize the toxicity of metformin among diabetic individuals.

\section{Acknowledgment}

This study was supported by a grant (2017-2018) from the University Grants Commission (UGC) of Bangladesh to A.A. Sajib. The authors are thankful to the UGC for its support.

\section{Statement of Ethics}

Buccal swab samples were collected from individuals upon receiving their consent. This study was approved by the Ethics Review Committee of the Faculty of Biological Sciences, University of Dhaka.

\section{Disclosure Statement}

The authors have no conflicts of interest to declare.

\section{Funding Sources}

This study was supported by a grant (2017-2018) from the University Grants Commission (UGC) of Bangladesh to A.A. Sajib.

\section{Author Contributions}

Idea and experiment design: A.A. Sajib. Experiment: T. Islam, M.S. Rahman, and A.A. Sajib. Evaluation of results and manuscript writing: N. Paul, S. Akhteruzzaman, and A.A. Sajib.

\section{References}

1 Hoogwerf BJ, Sferra J, Donley BG. Diabetes mellitus - overview. Foot Ankle Clin. 2006 Dec;11(4):703-15.

2 Ogurtsova K, da Rocha Fernandes JD, Huang Y, Linnenkamp U, Guariguata L, Cho NH, et al. IDF Diabetes Atlas: global estimates for the prevalence of diabetes for 2015 and 2040. Diabetes Res Clin Pract. 2017 Jun;128:40-50.

3 Chawla A, Chawla R, Jaggi S. Microvascular and macrovascular complications in diabetes mellitus: distinct or continuum? Indian J Endocrinol Metab. 2016 Jul-Aug;20(4):546-51.

4 Nyenwe EA, Jerkins TW, Umpierrez GE, Kitabchi AE. Management of type 2 diabetes: evolving strategies for the treatment of patients with type 2 diabetes. Metabolism. 2011 Jan;60(1):1-23.

5 American Diabetes Association. Diagnosis and classification of diabetes mellitus. Diabetes Care. 2014 Jan;37 Suppl 1:S81-90.

6 World Health Organization. WHO model list of essential medicines (20th list) [Internet] [Cited 2018 Oct 5]. Geneva: World Health Organization; 2017.

7 Gong L, Goswami S, Giacomini KM, Altman RB, Klein TE. Metformin pathways: pharmacokinetics and pharmacodynamics. Pharmacogenet Genomics. 2012 Nov;22(11):820-7.

Metformin Disposition: Allele-Specific Detection of rs316019 Variants
8 Luna B, Feinglos MN. Oral agents in the management of type 2 diabetes mellitus. Am Fam Physician. 2001 May;63(9):1747-56.

9 DeFronzo R, Fleming GA, Chen K, Bicsak TA. Metformin-associated lactic acidosis: current perspectives on causes and risk. Metabolism. 2016 Feb;65(2):20-9.

10 Fall PJ, Szerlip HM. Lactic acidosis: from sour milk to septic shock. J Intensive Care Med. 2005 Sep-Oct;20(5):255-71.

11 Friesecke S, Abel P, Roser M, Felix SB, Runge S. Outcome of severe lactic acidosis associated with metformin accumulation. Crit Care. 2010;14(6):R226.

12 Klachko D, Whaley-Connell A. Use of metformin in patients with kidney and cardiovascular diseases. Cardiorenal Med. 2011;1(2): 87-95.

13 Frid A, Sterner GN, Löndahl M, Wiklander C, Cato A, Vinge E, et al. Novel assay of metformin levels in patients with type 2 diabetes and varying levels of renal function: clinical recommendations. Diabetes Care. 2010 Jun; 33(6):1291-3.

14 Li Q, Liu F, Zheng TS, Tang JL, Lu HJ, Jia WP. SLC22A2 gene $808 \mathrm{G} / \mathrm{T}$ variant is related to plasma lactate concentration in Chinese type 2 diabetics treated with metformin. Acta Pharmacol Sin. 2010 Feb;31(2):184-90.
15 Piel S, Ehinger JK, Elmér E, Hansson MJ. Metformin induces lactate production in peripheral blood mononuclear cells and platelets through specific mitochondrial complex I inhibition. Acta Physiol (Oxf). 2015 Jan;213(1): 171-80.

16 Nies AT, Koepsell H, Damme K, Schwab M. Organic cation transporters (OCTs, MATEs), in vitro and in vivo evidence for the importance in drug therapy. In: Fromm M, Kim R, editors. Drug transporters. Handb Exp Pharmacol. Berlin/Heidelberg: Springer; 2011. Vol. 201; p. 105-67.

17 Koepsell H, Lips K, Volk C. Polyspecific organic cation transporters: structure, function, physiological roles, and biopharmaceutical implications. Pharm Res. 2007 Jul;24(7): 1227-51.

18 Viollet B, Guigas B, Sanz Garcia N, Leclerc J, Foretz M, Andreelli F. Cellular and molecular mechanisms of metformin: an overview. Clin Sci (Lond). 2012 Mar;122(6):253-70.

19 Song IS, Shin HJ, Kim WY, Lee CH, Shim JC, Zhou HH, et al. Identification and Functional Characterization of Genetic Variants of $\mathrm{Hu}$ man Organic Cation Transporters in a Korean Population. Drug Metab Dispos. 2007 Apr; 35(4):667-75. 
20 Song IS, Shin HJ, Shim EJ, Jung IS, Kim WY, Shon JH, et al. Genetic variants of the organic cation transporter 2 influence the disposition of metformin. Clin Pharmacol Ther. 2008 Nov;84(5):559-62.

21 Zolk O, Solbach TF, König J, Fromm MF. Functional characterization of the human organic cation transporter 2 variant p.270Ala[ $[\{G T\}]$ Ser. Drug Metab Dispos. 2009 Jun;37(6):1312-8.

22 Wilson NC, Choudhury A, Carstens N, Mavri-Damelin D. Organic cation transporter 2 (OCT2/SLC22A2) gene variation in the South African Bantu-speaking population and functional promoter variants. OMICS. 2017 Mar; 21(3):169-76.

23 Wang ZJ, Yin OQ, Tomlinson B, Chow MS. OCT2 polymorphisms and in-vivo renal functional consequence: studies with metfor$\mathrm{min}$ and cimetidine. Pharmacogenet Genomics. 2008 Jul;18(7):637-45.

24 Sajib AA, Islam T, Paul N, Yeasmin S. Interaction of rs316019 variants of SLC22A2 with metformin and other drugs - an in silico analysis. J Genet Eng Biotechnol. Epub ahead of print 2018 Feb 1.

25 Akter S, Rahman MM, Abe SK, Sultana P. Prevalence of diabetes and prediabetes and their risk factors among Bangladeshi adults: a nationwide survey. Bull World Health Organ. 2014 Mar;92(3):204-13.
26 Shariful Islam SM, Lechner A, Ferrari U, Laxy M, Seissler J, Brown J, et al. Healthcare use and expenditure for diabetes in Bangladesh. BMJ Glob Health. 2017 Jan;2(1):e000033.

27 Wangkumhang $\mathrm{P}$, Chaichoompu K, Ngamphiw C, Ruangrit U, Chanprasert J, Assawamakin A, et al. WASP: a Web-based AlleleSpecific PCR assay designing tool for detecting SNPs and mutations. BMC Genomics. 2007 Aug;8(1):275.

28 Ye J, Coulouris G, Zaretskaya I, Cutcutache I, Rozen S, Madden TL. Primer-BLAST: a tool to design target-specific primers for polymerase chain reaction. BMC Bioinformatics. 2012 Jun;13(1): 134.

291000 Genomes Project Consortium, Auton A, Brooks LD, Durbin RM, Garrison EP, Kang $\mathrm{HM}$, et al. A global reference for human genetic variation. Nature. 2015;526(7571):6874.

30 Hacker K, Maas R, Kornhuber J, Fromm MF, Zolk O. Substrate-dependent inhibition of the human organic cation transporter OCT2: a comparison of metformin with experimental substrates. PLoS One. 2015 Sep;10(9): e0136451.

31 Protti A, Russo R, Tagliabue P, Vecchio S, Singer M, Rudiger A, et al. Oxygen consumption is depressed in patients with lactic acidosis due to biguanide intoxication. Crit Care. 2010;14(1):R22.
32 Avery P, Mousa SS, Mousa SA. Pharmacogenomics in type II diabetes mellitus management: steps toward personalized medicine. Pharmgenomics Pers Med. 2009;2:79-91.

33 Twyman RM. Single nucleotide polymorphism (SNP) genotyping techniques - an overview. Encyclopedia of Diagnostic Genomics and Proteomics. New York, USA: Marcel Dekker, Inc; 2005. pp. 1202-7.

34 Rasmussen HB. Restriction fragment length polymorphism analysis of PCR-amplified fragments (PCR-RFLP) and gel electrophoresis - valuable tool for genotyping and genetic fingerprinting. Gel electrophoresis-principles and basics: IntechOpen. doi: 10.5772/37724.

35 Kwok PY, Chen X. Detection of single nucleotide polymorphisms. Curr Issues Mol Biol. 2003 Apr;5(2):43-60.

36 Latif ZA, Ashrafuzzaman S, Amin MF, Gadekar AV, Sobhan MJ, Haider T. A Crosssectional Study to Evaluate Diabetes Management, Control and Complications in Patients with type 2 Diabetes in Bangladesh. BIRDEM Med J. 2017;7(1):17-27.

37 Lek M, Karczewski KJ, Minikel EV, Samocha KE, Banks E, Fennell T, et al.; Exome Aggregation Consortium. Analysis of protein-coding genetic variation in 60,706 humans. $\mathrm{Na}$ ture. 2016 Aug;536(7616):285-91. 